\title{
EMPIRIC INTRAVENOUS ANTIBOTIC THERAPY ON GASTROENTERITIS UP ON CLINICAL AND STOOL EXAM EVIDENCES
}

\author{
M. Fatemi Khorasgani, E. Gholamian, M. Moslehi
}

Pediatrics, Islamic Azad University- Najafabad branch-Shariati Hospital, Isfahan, Iran

Introduction: Gastroenteritis is endemic in many countries. Intravenous antibiotic therapy is a challenge due to difficulties of outpatient diagnosis and laboratory evaluation. This study is about the effect of empiric antibiotic therapy on duration of stay in hospital in patients up on PMN findings in stool exam.

Materials and methods: 201 patients between 6 months to 5 years(mean age 20 months), 112 male and 89 female, with gastroenteritis in pediatric ward of Shariati hospital in Isfahan from May to December 2010 take place in study. Their treatment methodology was same and everyone should have stool exam although 40 didn't do it (Missing group). Another groups are patients with 0-5 PMN and more than 5 PMN in stool exam. We used SPSS ver. 15 ,Levene's Test and t-test for evaluation.

Results: In $\mathrm{PMN}=0-5$ group(120 patients), mean time of stay for 51 who received antibiotics were 104.47 hours and for 69 without antibiotics were 61.91 , so with P-value $<0.005$ therapy lengthened stay time. In $\mathrm{PMN}>5$ group(41 patients), mean time for 3 without antibiotics 48 hours and for 38 with antibiotics were 89.66 that with P-value $<0.005$ antibiotic group had more stay time. In Missed group(40 without stool exam) 10 with antibiotics had mean time of 64.80 and 30 others 48.80 , with equally assumed variances(sig. $=0.005$ ). Average time for antibiotic group(99) were 94.78 and for 102 without antibiotics 94.78 hours with significant time length (P-value $<0.005)$.

Conclusion: IV antibiotic therapy base on only clinical evidences and stool exam length time of patient admission in gastroenteritis. 